\title{
An EOQ Model for Perishable Items with Supply Uncertainty
}

\author{
Xiaoming Yan ${ }^{1,2}$ and Yong Wang ${ }^{3}$ \\ ${ }^{1}$ School of Computer, Dongguan University of Technology, Dongguan, Guangdong 523808, China \\ ${ }^{2}$ Chongqing Key Laboratory of Logistics, Chongqing University, Chongqing 400044, China \\ ${ }^{3}$ School of Economics and Business Administration, Chongqing University, Chongqing 400044, China
}

Correspondence should be addressed to Yong Wang; wangyongkt@126.com

Received 10 December 2012; Accepted 31 January 2013

Academic Editor: Sivaguru Ravindran

Copyright (c) 2013 X. Yan and Y. Wang. This is an open access article distributed under the Creative Commons Attribution License, which permits unrestricted use, distribution, and reproduction in any medium, provided the original work is properly cited.

\begin{abstract}
This paper aims to develop a continuous inventory model for finding the strategy for a retailer that sells a seasonal perishable item over a finite planning time. The purpose of this retailer is to maximize the expected profit by choosing an optimal ordering quantity before the sales horizon and adopting an optimal pricing policy during the sales horizon. With the help of Pontryagin's maximum principle, we can obtain the optimal ordering quantity and the optimal pricing policy by solving a list of equations. For a special case, we not only characterize the structure of the optimal pricing policy for any given initial inventory level but also obtain the optimal solutions by solving a set of equations. A numerical analysis reveals the influence of some parameters on the optimal ordering quantity.
\end{abstract}

\section{Introduction}

Many unpredictable events, such as machine breakdowns, unscheduled maintenance, labor strikes, and natural disaster, exist widely in the previous several decades and produce some adverse and long-lasting effects on the downstream supply chain members. For example, in March 2000, lightning caused a fire that shut down the Philips Semiconductor plant in Albuquerque, New Mexico, for six weeks, leading to a shortage of components for both Ericsson and Nokia. According to The Wall Street Journal, company officials say that they [Ericsson] lost at least 400 million in potential revenue [1]. Moreover, we can find a large number of other examples in Sheffi's book [2]. The wide existence of yield uncertainty has drawn significant attention of scholars and turns the random yield to an important research topic in the area of operations management and production economics. For example, Gerchak et al. [3], Ciarallo et al. [4], Wang and Gerchak [5], Erdem and Özekici [6], Li and Zheng [7], Chao et al. [8], and Feng [9] analyze the inventory systems with random yield and periodic review; Erdem et al. [10], Fadıloğlu et al. [11], Yan and Liu [12], and Tajbakhsh et al. [13] address the random yield models with continuous review; Agrawal and Nahmias [14], Inderfurth [15], Dada et al. [16], Grasman et al. [17], Rekik et al. [18], Maddah et al. [19], and Tang and Kouvelis [20] study the newsvendor problems with unreliable supply and uncertain demand.

In this paper, we consider an EOQ model where a retailer orders, stocks, and sells a perishable item over a fixed finite horizon. Before the sales horizon, the retailer orders a quantity of an item which can be received at the beginning of the sales horizon. However, the supplier may be unreliable, that is, the quantity effectively received at the beginning of the sales horizon cannot correspond exactly to the ordering quantity. After the ordering quantity has been received and the sales horizon has started, the retailer sells the item by adopting a dynamic pricing policy. We allow the prices to be varied continuously over the sales horizon and the item to be of physical decay or deterioration. Deterioration of inventory is commonplace in real life, such as fruit, vegetables, and seafood. At the end of the sales horizon, the inventory, which is not sold during the horizon, will be disposed of with a salvage, which may be negative. Once the products have been sold out before the end of the horizon, the retailer stops the selling behavior and the trade is over. The objective of the retailer is to maximize his expected profit by determining an ordering quantity before the horizon and adopting a dynamic pricing policy during the horizon. This problem arises in 
a variety of industries, such as supermarket selling vegetables, fruits, and fishes.

Next, we review some literatures on EOQ models with pricing decisions. Rajan et al. [21] consider the relationship between pricing and ordering decisions for a monopolistic retailer facing a known demand function which is deterministic. They achieve the optimal pricing policy and the length of pricing cycle. Abad [22] extends Rajan et al. [21]'s model, and the only difference is that when it is economic for a highly perishable product to backlog demand, the retailer can plan for periods of shortage during which demand can be partially backlogging. Abad [23] considers the problem of determining the optimal price and lot size for a reseller, where the selling price is constant within the inventory cycle. Teng and Chang [24] establish an EPQ model in which the demand rate is a function of the on-display stock level and the selling price. J. M. Chen and L. T. Chen [25] deal with the joint decisions on pricing and replenishment schedule for a periodic review inventory system in which a replenishment order may be placed at the beginning of some or all of the periods. Shinn and Hwang [26] deal with the problem of determining the retailer's optimal price and order size simultaneously under the condition of order-size-dependent delay in payments. Chou and Parlar [27] consider a dynamic pricing problem over a finite horizon where the demand for a product is a time-varying linear function of price. Our work is most closely related to the model considered by You and Hsieh [28]. They study an EOQ model for a nonperishable product with stock- and price-sensitive demand. In their model, the price is varied periodically and the supplier is reliable. However, we study an EOQ model with random yield and continuously dynamic pricing decisions. By transforming the primal problem into a standard control problem, the optimal pricing policy can be obtained by solving a list of equations. To a certain extent, our model is an extension of You and Hsieh's model. Many other literatures on dynamic pricing model can be found in the review papers, such as Chan et al. [29] and Elmaghraby and Keskinocak [30].

The rest of this paper is organized as follows. In Section 2, we present a mathematical model and transform the retailer's problem into a standard control problem. In Section 3, we determine the optimal pricing policy and initial ordering quantity for a special case. In Section 4, we present some conclusions and directions for future research.

\section{Mathematical Model and Analysis}

Consider a retailer which orders and sells a perishable product over a finite time horizon. Before the sales horizon, the retailer orders a quantity of an item which can be received at the beginning of the sales horizon. However, the quantity effectively received at the beginning of the sales horizon may be less than the ordering quantity. After the ordering quantity has been received and the sales horizon has started, the retailer sells the item by adopting a dynamic pricing policy. In our formulation, let $w$ be the unit purchasing cost, $h$ the per unit inventory holding cost per unit time, $\theta$ the discount rate, $T$ the horizon length, $v$ the unit salvage value at the end of the horizon, and $q$ the ordering quantity before the sales horizon. However, an order placed from the supplier may be realized randomly: an ordering of $q$ units from the supplier will receive $\xi q$ units of the item at the beginning of the horizon, where $\xi$ is a random variable whose distribution is independent of the ordering quantity $q . \Psi(\cdot)$ and $\varphi(\cdot)$ denote the distribution and density functions of $\xi$, respectively. Without loss of generality, we assume that $0 \leq \xi \leq 1$ with probability one and $\varphi(z)>0$ for $0 \leq z \leq 1$. This form of random yield has been used by many other researchers, such as Gerchak et al. [3], Grasman et al. [17], Inderfurth [15], Agrawal and Nahmias [14], and Li and Zheng [7]. Let $I(t)$ be the inventory level at time $t$. Because we assume that the retailer stops selling once he/she sells out the item, we can derive $I(t) \geq 0$ for all $t \in[0, T]$. The deterioration rate at time $t$ is assumed to be $W(t)=a(t) I(t)$, where $a(t) \geq 0$ is the deterioration factor at time $t$. If $a(t)=0$, then there is no deterioration at time $t$. Let $p(t) \geq 0$ be the unit selling price at time $t \in[0, T]$. The demand rate at time $t$, denoted as $D(p(t), t)$, is a function both of price $p(t)$ and time $t$. Because the customers often prefer to purchase fresher and cheaper items, we assume that the demand rate, $D(p(t), t)$, is nonincreasing with $p(t)$ and $t$, respectively. Let $\bar{p}(t)$ be the finite upper bound of price $p(t)$, that is, $D(\bar{p}(t), t)=0$ for $t \in[0, T]$. Therefore, $p(t)=\bar{p}(t)$ denotes that the item has been sold out and the trade is over.

If the retailer picks a pricing policy $p(t)$ for $t \in[0, T]$, then the total demand during $[0, T]$ can be described as $\int_{0}^{T} D(p(t), t) d t$, and the total deterioration during $[0, T]$ can be described as $\int_{0}^{T} W(t) d t$. Therefore, for any fixed inventory level $Q$ at the beginning of the horizon, we have the following constraint:

$$
\int_{0}^{T}[D(p(t), t)+W(t)] d t \leq Q .
$$

In fact, $Q$ denotes the realized value of the ordering quantity at the beginning of the horizon. Constraint (1) states that the total of demand and deterioration during the horizon is never more than the initial inventory level $Q$.

For any initial inventory level $Q$ and pricing policy $p(\cdot)$, the retailer's profit obtained during the sales horizon, $J(p(\cdot), Q)$, is given by the sales revenue over the horizon, adding the salvage profit and subtracting the holding cost over the horizon

$$
\begin{aligned}
& J(p(\cdot), Q) \\
& \quad=\int_{0}^{T} e^{-\theta t}[p(t) D(p(t), t)-h I(t)] d t+v e^{-\theta T} I(T),
\end{aligned}
$$

where the inventory level, $I(t)$, satisfies the following condition

$$
d I(t)=[-D(p(t), t)-a(t) I(t)] d t, \quad I(0)=\mathrm{Q} .
$$

By solving (3), the inventory level, $I(t)$, can be described as

$$
I(t)=e^{-\int_{0}^{t} a(u) d u}\left[Q-\int_{0}^{t} e^{\int_{0}^{s} a(u) d u} D(p(s), s) d s\right] .
$$


Expression (4) implies that the inventory level at time $T$ is

$$
I(T)=e^{-\int_{0}^{T} a(u) d u}\left[Q-\int_{0}^{T} e^{\int_{0}^{s} a(u) d u} D(p(s), s) d s\right] .
$$

Based on (4) and (5), we derive the following result which presents the closed form of $J(p(\cdot), Q)$. All proofs are presented in the appendix.

Proposition 1. $J(p(\cdot), Q)$, defined as (2), can be simplified as

$$
J(p(\cdot), Q)=A+\int_{0}^{T} e^{-\theta t}[p(t)-c(t)] D(p(t), t) d t
$$

where $A$ and $c(t)$ are defined as follows:

$$
\begin{gathered}
A=v Q e^{-\theta T-\int_{0}^{T} a(u) d u}-h Q \int_{0}^{T} e^{-\theta t-\int_{0}^{t} a(u) d u} d t, \\
c(t)=v e^{-\theta(T-t)-\int_{t}^{T} a(u) d u}-h \int_{t}^{T} e^{\theta(t-s)-\int_{t}^{s} a(u) d u} d s .
\end{gathered}
$$

Note that $A$, defined as (7), is independent of the pricing policy $p(\cdot)$; therefore, for any given initial inventory level $Q$, the retailer's problem during the sales horizon can be equivalently described as follows:

$$
\max _{0 \leq p(\cdot) \leq \bar{p}(\cdot)} \int_{0}^{T} e^{-\theta t}[p(t)-c(t)] D(p(t), t) d t,
$$

s.t.

$$
0 \leq I(t) \leq Q, \quad I(t)=0, \forall t \geq \tau
$$

where $\tau=\min \{0 \leq t \leq T: I(t)=0\}$. If $\{0 \leq t \leq T: I(t)=$ $0\}=\varnothing$, then $I(T)>0$ and $\tau \triangleq+\infty$, that is, part of the items are unsold at time $T$. Therefore, if $\tau<T$, then $\tau$ denotes the time at which the retailer just sells out the items and the trade is over at time $\tau$. If $\tau=T$, then the retailer just sells out the items at time $T$. If $\tau=+\infty$, then part of the items are unsold at time $T$. From the expression of $I(t)$ in (4), constraint (10) can be equivalently written as

$$
\begin{gathered}
\int_{0}^{t} e^{\int_{0}^{s} a(u) d u} D(p(s), s) d s \leq Q, \quad \forall 0 \leq t \leq T, \\
\int_{0}^{t} e^{\int_{0}^{s} a(u) d u} D(p(s), s) d s=Q, \quad \forall t \geq \tau .
\end{gathered}
$$

In order to solve the optimization problem (9), we try to transform it into a standard control problem. For notational convenience, we define

$$
y(t)=\int_{0}^{t} D(p(s), s) e^{\int_{0}^{s} a(u) d u} d s .
$$

Note that $0 \leq p(t) \leq \bar{p}(t)$, we have $D(p(t), t) \geq 0$ and $y(t)$ is increasing in $t$. Therefore, the problem (9) can be transformed into the following standard control problem:

$$
\max _{0 \leq p(\cdot) \leq \bar{p}(\cdot)} \int_{0}^{T} e^{-\theta t}[p(t)-c(t)] D(p(t), t) d t,
$$

s.t.

$$
y(0)=0, \quad y(T) \leq Q, \quad y^{\prime}(t)=D(p(t), t) e^{\int_{0}^{t} a(u) d u}
$$

where $p(t)$ is regarded as a control variable and $y(t)$ is regarded as a state variable. According to Sethi and Thompson (Section 3.3, page 65, [31]), the current-value Hamiltonian function with adjoint variable $\lambda$ can be described as

$$
H(p, \lambda, t)=[p-c(t)] D(p, t)+\lambda D(p, t) e^{\int_{0}^{t} a(u) d u}
$$

and the current-value Lagrangian function with Lagrange multipliers $\mu_{1}$ and $\mu_{2}$ can be described as

$$
L\left(p, \lambda, \mu_{1}, \mu_{2}, t\right)=H(p, \lambda, t)+\mu_{1} p+\mu_{2}(\bar{p}-p),
$$

where $\lambda, \mu_{1}, \mu_{2}, p$ and $\bar{p}$ are all dependent on time $t$. Following Theorem 8 and expression (3.41) in Sethi and Thompson [31], we present the following result.

Lemma 2. If $H(p, \lambda, t)$ is concave in $p$, then according to Pontryagin's maximum principle, the optimal control $p^{*}(t)$ must satisfy the following necessary and sufficient conditions:

$$
\begin{gathered}
\left.\frac{\partial L\left(p, \lambda, \mu_{1}, \mu_{2}, t\right)}{\partial p}\right|_{p=p^{*}} \\
=D\left(p^{*}, t\right)+\left[p^{*}-c(t)+\left.\lambda e^{\left.\int_{0}^{t} a(u) d u\right]} \frac{\partial D(p, t)}{\partial p}\right|_{p=p^{*}}\right. \\
+\mu_{1}-\mu_{2}=0, \\
\lambda^{\prime}=\theta \lambda-\frac{\partial H\left(p^{*}, t, \lambda\right)}{\partial y}=\theta \lambda, \quad \lambda(T) \leq 0 \\
y(T) \leq Q, \quad \lambda(T)[y(T)-Q]=0 \\
0 \leq p^{*} \leq \bar{p}, \quad \mu_{1} p^{*}=\mu_{2}\left(\bar{p}-p^{*}\right)=0 \\
H\left(p^{*}, \lambda, t\right) \geq H(p, \lambda, t), \quad \mu_{1} \geq 0, \quad \mu_{2} \geq 0
\end{gathered}
$$

Based on Lemma 2, the optimal price at time $t$ is equivalently written as $p^{*}(t)=\arg \max _{0 \leq p \leq \bar{p}(t)}\{H(p, \lambda(t), t)\}$, where $\lambda(t)$ is determined by (17). For convenience in exposition, we define

$$
\begin{aligned}
\widetilde{p}(t, \lambda) & \\
= & 0 \vee \arg \max _{p}\left\{\left[p-c(t)+\lambda e^{-\theta(T-t)} e^{\int_{0}^{t} a(u) d u}\right] D(p, t)\right\} \\
& \wedge \bar{p}(t),
\end{aligned}
$$

where $x_{1} \wedge x_{2}=\min \left\{x_{1}, x_{2}\right\}$ and $x_{1} \vee x_{2}=\max \left\{x_{1}, x_{2}\right\}$. The following proposition characterizes the optimal pricing policy. 
Proposition 3. If $H(p, \lambda, t)$ is concave in $p$, then the optimal pricing policy can be described as follows.

(1) If $\int_{0}^{T} D(\widetilde{p}(s, 0), s) e^{\int_{0}^{s} a(u) d u} d s \leq Q$, then $\widetilde{p}(t, 0)$ is just the optimal pricing policy.

(2) If $\int_{0}^{T} D(\tilde{p}(s, 0), s) e^{\int_{0}^{s} a(u) d u} d s>Q$, then the optimal pricing policy is given by $\widetilde{p}(t, \lambda(T))$, where $\lambda(T)$ is determined by

$$
\int_{0}^{T} D(\widetilde{p}(s, \lambda(T)), s) e^{\int_{0}^{s} a(u) d u} d s=Q
$$

Proposition 3 implies that the optimal pricing policy is determined by the realized initial inventory level $Q$. If $Q$ is large enough (i.e., $\left.Q \geq \int_{0}^{T} D(\widetilde{p}(s, 0), s) e^{\int_{0}^{s} a(u) d u} d s\right)$, then the optimal pricing policy can be described as $0 \vee \arg \max _{p}\{[p-$ $c(t)] D(p, t)\} \wedge \bar{p}(t)$. Otherwise, the optimal pricing policy is dependent on the value of $\lambda(T)$, which can be determined by solving (20). Since the optimal pricing policy is dependent on the initial inventory level, $Q$, we denote the optimal pricing policy as $p^{*}(\cdot, Q)$ for given initial inventory level $Q$. After the optimal pricing policy, $p^{*}(\cdot, Q)$, has been obtained for any initial inventory level $Q$, the retailer determines the optimal ordering quantity $q^{*}$ according to the following expression:

$$
q^{*}=\arg \max _{q \geq 0}\left\{E J\left(p^{*}(\cdot, q \xi), q \xi\right)-w q E \xi\right\},
$$

where $\xi_{q}$ denotes the realized initial inventory level at the beginning of the sales horizon. In expression (21), we assume that the retailer pays for the quantity effectively received, and the retailer is risk neutral. The results can be extended to the case in which the retailer pays for the ordering quantity.

\section{An Important Case}

While the general formulation discussed in the above section is useful to obtain the optimal solution for the complex real world application, we can gain stronger results and insights into the problem for specific cases. In this section, we mainly concentrate on analyzing a special case in which the demand rate at time $t$ is

$$
D(p(t), t)=\alpha-\beta e^{\mu t} p(t),
$$

where $\alpha \geq 0, \beta \geq 0$ and $\mu \geq 0$ are constants. In expression (22), $\mu \geq 0$ implies that if $p(t)=p$ for all $t \in[0, T]$, then the demand rate is decreasing in $t$. That is, if the price keeps constant during the sales horizon, the customers always prefer to purchase fresher items. Demand (22) is derived from the linear demand form: $d(p)=\alpha-\beta p$, which has been used by many researchers, such as Chao et al. [8]. From (22), we derive $\bar{p}(t)=(\alpha / \beta) e^{-\mu t}$. In what follows, we assume $a(t)=a$ for all $0 \leq t \leq T$, where $a$ is a nonnegative number. In order to avoid trivial cases, we present the following assumption which always holds in the following analysis.

Assumption 4. Consider the following:

$$
v e^{-(a+\theta) T}<w+\frac{h}{\theta+a}\left[1-e^{-(\theta+a) T}\right]<\frac{\alpha}{\beta} e^{-(a+\theta+\mu) T} .
$$

Remark 5. From (4), the holding cost of storing unit item from time zero to $T$ can be described as

$$
h \int_{0}^{T} e^{-\theta t} \cdot 1 \cdot e^{-a t} d t=\frac{h}{\theta+a}\left[1-e^{-(\theta+a) T}\right],
$$

where $e^{-a t}$ is the leavings of unit product at time $t$. Therefore, the first inequality of Assumption 4 states that it is not profitable for the retailer to purchase more products for disposing with salvage $v$ at the end of the horizon, and the second inequality of Assumption 4 states that it is profitable to purchase products for selling at the end of the sales horizon.

3.1. Optimal Pricing Policy. Note that $D(p(t), t)=\alpha-\beta e^{\mu t} p(t)$ and $a(t)=a$ for $0 \leq t \leq T$, then from (15), (7), and (8), the current-value Hamilton function $H(p, \lambda, t), A$ and $c(t)$ can be reduced to the following expressions:

$$
\begin{gathered}
H(p, \lambda, t)=\left[p-c(t)+\lambda e^{a t}\right]\left(\alpha-\beta e^{\mu t} p\right), \\
A=v Q e^{-(\theta+a) T}-\frac{h Q}{\theta+a}\left[1-e^{-(\theta+a) T}\right], \\
c(t)=v e^{-(\theta+a)(T-t)}-\frac{h}{\theta+a}\left[1-e^{-(\theta+a)(T-t)}\right] .
\end{gathered}
$$

Taking second-order partial derivative of $H(p, \lambda, t)$ with respect to $p$, we have

$$
\frac{\partial^{2} H(p, \lambda, t)}{\partial p^{2}}=-2 \beta e^{\mu t}<0
$$

That is, $H(p, \lambda, t)$ is strictly concave in $p$. Therefore, we can obtain the optimal pricing policy by Proposition 3. From $\lambda(t)=\lambda(T) e^{-\theta(T-t)}$ and expression (25), $\partial H(p, \lambda, t) / \partial p=0$ can be written as

$$
\alpha-\beta e^{\mu t} p(t)-\left[p(t)-c(t)+\lambda(T) e^{a t-\theta(T-t)}\right] \beta e^{\mu t}=0 .
$$

By solving above equation, we derive

$$
\widetilde{p}(t, \lambda(T))=\frac{\alpha+\beta e^{\mu t} c(t)-\beta \lambda(T) e^{(a+\theta+\mu) t-\theta T}}{2 \beta e^{\mu t}} .
$$

From (30) and $0 \leq p^{*}(t) \leq(\alpha / \beta) e^{-\mu t}$, the optimal pricing policy can be described as

$$
\begin{aligned}
& \widetilde{p}(t, \lambda(T)) \\
& \quad=\min \left\{\max \left\{\frac{\alpha+\beta e^{\mu t} c(t)-\beta \lambda(T) e^{(a+\theta+\mu) t-\theta T}}{2 \beta e^{\mu t}}, 0\right\}, \frac{\alpha}{\beta} e^{-\mu t}\right\} .
\end{aligned}
$$

Note that $c(t)$ is defined as (27), then after simplifying, the optimal pricing policy can be written as

$$
\widetilde{p}(t, \lambda(T))=\min \left\{\max \{f(t, \lambda(T)), 0\}, \frac{\alpha}{\beta} e^{-\mu t}\right\},
$$


where

$$
\begin{array}{r}
f(t, \lambda(T))=\frac{\alpha}{2 \beta} e^{-\mu t}+B(\lambda(T)) e^{(\theta+a) t}-\frac{h}{2(\theta+a)}, \\
B(\lambda(T))=\frac{v}{2} e^{-(\theta+a) T}+\frac{h}{2(\theta+a)} e^{-(\theta+a) T}-\frac{\lambda(T)}{2} e^{-\theta T} .
\end{array}
$$

In reality, the selling price of any product is always nonnegative. Therefore, we assume that $f(t, \lambda(T)) \geq 0$ for any $t \in[0, T]$ and $\lambda(T) \leq 0$, otherwise, there exists some time interval on which the optimal price is zero. That is, we assume that the parameters $(\alpha, \beta, \mu, \theta, a, h, v$, and $T)$ obtained from the real life guarantee $f(t, \lambda(T)) \geq 0$ for any $t \in[0, T]$ and $\lambda(T) \leq 0$. From (33), $f(t, \lambda(T)) \geq 0$ can be written as

$$
\begin{aligned}
\frac{\alpha}{2 \beta} e^{-\mu t}+ & {\left[\frac{v}{2} e^{-(\theta+a) T}+\frac{h}{2(\theta+a)} e^{-(\theta+a) T}-\frac{\lambda(T)}{2} e^{-\theta T}\right] e^{(\theta+a) t} } \\
- & \frac{h}{2(\theta+a)} \geq 0 .
\end{aligned}
$$

Since $\lambda(T) \leq 0$ in the optimal situation, expression (34) is equivalent to $g(t) \geq 0$ for $t \in[0, T]$, where

$$
\begin{aligned}
g(t)= & \frac{\alpha}{2 \beta} e^{-\mu t}+\left[\frac{v}{2} e^{-(\theta+a) T}+\frac{h}{2(\theta+a)} e^{-(\theta+a) T}\right] e^{(\theta+a) t} \\
& -\frac{h}{2(\theta+a)} .
\end{aligned}
$$

The following result presents several conditions under which $g(t) \geq 0$ for $t \in[0, T]$.

Lemma 6. If $t_{0} \leq 0$ or $t_{0} \geq T$, then $g(t)>0$ for $t \in[0, T]$; if $0<t_{0}<T$ and $g\left(t_{0}\right) \geq 0$, then $g(t) \geq 0$ for $t \in[0, T]$, where $t_{0}$ and $g\left(t_{0}\right)$ are defined as (A.8) and (A.9), respectively.

Based on $\lambda(T) \leq 0$, Assumption 4 and $g(t) \geq 0$ for $t \epsilon$ $[0, T]$, we present the following result which formulates the relationship between the value of $\lambda(T)$ and the structure of the optimal pricing policy.

Lemma 7. (1) If

$$
\min \left\{v e^{-a T}-\frac{\alpha}{\beta} e^{-(a+\mu) T}, 0\right\}<\lambda(T) \leq 0,
$$

then the optimal pricing policy can be described as $\widetilde{p}(t, \lambda(T))=$ $f(t, \lambda(T))$ for $t \in[0, T]$.

(2) If

$$
\begin{gathered}
-\frac{\alpha}{\beta} e^{\theta T}+v e^{-a T}-\frac{h}{\theta+a}\left(e^{\theta T}-e^{-a T}\right) \\
<\lambda(T) \leq \min \left\{v e^{-a T}-\frac{\alpha}{\beta} e^{-(a+\mu) T}, 0\right\},
\end{gathered}
$$

then the optimal pricing policy can be described as $\widetilde{p}(t, \lambda(T))=$ $f(t, \lambda(T))$ for $t \in[0, t(\lambda(T))]$ and $\tilde{p}(t, \lambda(T))=(\alpha / \beta) e^{-\mu t}$ for $t \in(t(\lambda(T)), T]$, where $t(\lambda(T))$ satisfies (A.12).

(3) If

$$
\lambda(T) \leq-\frac{\alpha}{\beta} e^{\theta T}+v e^{-a T}-\frac{h}{\theta+a}\left(e^{\theta T}-e^{-a T}\right),
$$

then the optimal pricing policy can be described as $\widetilde{p}(t, \lambda(T))=$ $(\alpha / \beta) e^{-\mu t}$ for $t \in[0, T]$, that is, the retailer will not order and sell the product in the optimal situation.

Lemma 7 states that the optimal pricing policy can be determined by the value of $\lambda(T)$. That is, once the value of $\lambda(T)$ has been determined, the optimal pricing policy is also obtained. In order to guarantee that it is better to sell the product during the horizon than to salvage the product at the end of the horizon, we assume that $v<(\alpha / \beta) e^{-\mu T}$ in the rest of this paper. Next, we want to determine the optimal value of $\lambda(T)$. For notational convenience, we define

$$
\begin{aligned}
\gamma_{1}(x)= & \int_{0}^{x}\left(\frac{\alpha}{2} e^{a t}-\frac{\beta}{2} e^{(a+\mu) t} c(t)\right) d t \\
= & \frac{\alpha}{2 a}\left(e^{a x}-1\right)-\frac{\beta v(\theta+a) e^{-(\theta+a) T}+\beta h e^{-(\theta+a) T}}{2(\theta+a)(2 a+\theta+\mu)} \\
& \times\left[e^{(2 a+\theta+\mu) x}-1\right]+\frac{\beta h}{2(\theta+a)(a+\mu)}\left[e^{(a+\mu) x}-1\right], \\
\gamma_{2}(x)= & \int_{0}^{x} \frac{\beta}{2} e^{(2 a+\theta+\mu) t-\theta T} d t \\
= & \frac{\beta e^{-\theta T}}{2(2 a+\theta+\mu)}\left(e^{(2 a+\theta+\mu) x}-1\right),
\end{aligned}
$$

$$
\begin{aligned}
\gamma_{3}(x)= & \int_{0}^{T} e^{-\theta t}\left\{\frac{e^{-\mu t}}{\beta}\left[\frac{\alpha}{2}+\frac{\beta}{2} e^{\mu t} c(t)\right]^{2}-\alpha c(t)\right\} d t \\
= & \int_{0}^{x}\left\{\frac{\beta}{4} e^{(\mu-\theta) t} c^{2}(t)-\frac{\alpha}{2} e^{-\theta t} c(t)+\frac{\alpha^{2}}{4 \beta} e^{-(\theta+\mu) t}\right\} d t \\
= & \frac{1}{2 a+\theta+\mu}\left[\frac{\beta v^{2}}{4}+\frac{\beta h^{2}}{4(\theta+a)^{2}}+\frac{\beta v h}{2(\theta+a)}\right] \\
& \times\left[e^{(2 a+\theta+\mu) x}-1\right] e^{-2(\theta+a) T} \\
& -\frac{1}{a+\mu}\left[\frac{\beta h^{2}}{2(\theta+a)^{2}}+\frac{\beta v h}{2(\theta+a)}\right]\left[e^{(a+\mu) x}-1\right] e^{-(\theta+a) T} \\
& +\frac{\beta h^{2}}{4(\theta+a)^{2}(\mu-\theta)}\left[e^{(\mu-\theta) x}-1\right] \\
& -\left[\frac{\alpha v}{2 a}+\frac{\alpha h}{2 a(\theta+a)}\right]\left(e^{a x}-1\right) e^{-(\theta+a) T} \\
& +\frac{\alpha h}{2 \theta(a+\theta)}\left(1-e^{-\theta x}\right)+\frac{\alpha^{2}}{4 \beta(\theta+\mu)}\left[1-e^{-(\theta+\mu) x}\right] .
\end{aligned}
$$


In expression (40), we assume that $\mu-\theta \neq 0$. If $\mu-\theta=$ 0 , then we set $(1 /(\mu-\theta))\left[e^{(\mu-\theta) x}-1\right]=0$ in expression (40). From expression (A.6), we obtain that the optimal value of $\lambda(T)$ is dependent on the initial inventory level. Note that the optimal pricing policy can be determined by the value of $\lambda(T)$, therefore, the optimal pricing policy can be determined by the initial inventory level. Because the initial inventory level is fixed at the beginning of the sales horizon, we can determine the optimal value of $\lambda(T)$ by analyzing the relationship between the value of $\lambda(T)$ and the initial inventory level. The next result formulates the optimal value of $\lambda(T)$ and the structure of the optimal pricing policy based on the initial inventory level $Q$.

Theorem 8. (1) If $Q>Q_{0}$, then $\lambda(T)=0$, and the optimal pricing policy can be described as

$$
\begin{aligned}
p^{*}(t, Q)= & \frac{\alpha+\beta e^{\mu t} c(t)}{2 \beta e^{\mu t}}=\frac{\alpha}{2 \beta} e^{-\mu t} \\
& +\left[\frac{v}{2} e^{-(\theta+a) T}+\frac{h}{2(\theta+a)} e^{-(\theta+a) T}\right] e^{(\theta+a) t} \\
& -\frac{h}{2(\theta+a)}
\end{aligned}
$$

(2) If $Q_{1} \leq Q \leq Q_{0}$, then $\lambda(T)=\left(Q-\gamma_{1}(T)\right) / \gamma_{2}(T)$, and the optimal pricing policy can be described as

$$
\begin{array}{r}
P^{*}(t, Q) \\
=\frac{\alpha+\beta e^{\mu t} c(t)-\left(\left(Q-\gamma_{1}(T)\right) / \gamma_{2}(T)\right) \beta e^{(a+\theta+\mu) t-\theta T}}{2 \beta e^{\mu t}} \\
=\frac{\alpha}{2 \beta} e^{-\mu t}+\left[\frac{v}{2} e^{-(\theta+a) T}+\frac{h}{2(\theta+a)} e^{-(\theta+a) T}\right. \\
\left.-\frac{Q-\gamma_{1}(T)}{2 \gamma_{2}(T)} e^{-\theta T}\right] e^{(\theta+a) t}-\frac{h}{2(\theta+a)} .
\end{array}
$$

(3) If $Q<Q_{1}$, then $\lambda(T)=\left(Q-\gamma_{1}(t(Q))\right) / \gamma_{2}(t(Q))$, and the optimal pricing policy can be described as

$$
\begin{array}{r}
p^{*}(t, Q) \\
=\frac{\alpha+\beta e^{\mu t} c(t)-\left(\left(Q-\gamma_{1}(t(Q))\right) / \gamma_{2}(t(Q))\right) \beta e^{(a+\theta+\mu) t-\theta T}}{2 \beta e^{\mu t}} \\
=\frac{\alpha}{2 \beta} e^{-\mu t}+\left[\frac{v}{2} e^{-(\theta+a) T}+\frac{h}{2(\theta+a)} e^{-(\theta+a) T}\right. \\
\left.\quad-\frac{Q-\gamma_{1}(t(Q))}{2 \gamma_{2}(t(Q))} e^{-\theta T}\right] e^{(\theta+a) t}-\frac{h}{2(\theta+a)}
\end{array}
$$

for $0 \leq t \leq t(Q)$ and $p^{*}(t, Q)=(\alpha / \beta) e^{-\mu t}$ for $t(Q)<t \leq T$, where $t(Q), Q_{0}$, and $Q_{1}$ are defined as

$$
\begin{aligned}
Q= & \gamma_{1}(t(Q))+\gamma_{2}(t(Q)) \\
& \times\left[v e^{-a T}+\frac{h}{\theta+a} e^{-a T}-\frac{\alpha}{\beta} e^{\theta T-(a+\theta+\mu) t(Q)}\right. \\
& \left.-\frac{h}{\theta+a} e^{\theta T-(\theta+a) t(Q)}\right], \\
Q_{0}= & \frac{\alpha}{2 a}\left(e^{a T}-1\right)-\frac{\beta v}{2(2 a+\theta+\mu)}\left[e^{(a+\mu) T}-e^{-(\theta+a) T}\right] \\
+ & \frac{\beta h}{2(\theta+a)(a+\mu)}\left[e^{(a+\mu) T}-1\right] \\
- & \frac{\beta h}{2(\theta+a)(2 a+\theta+\mu)}\left[e^{(a+\mu) T}-e^{-(a+\theta) T}\right], \\
Q_{1} & =Q_{0}-\frac{\alpha-\beta v e^{\mu T}}{2(2 a+\theta+\mu)}\left[e^{a T}-e^{-(a+\theta+\mu) T}\right] .
\end{aligned}
$$

Theorem 8 implies that the structure of the optimal pricing policy can be determined by the initial inventory level. If the initial inventory level $Q$ is more than $Q_{0}$, that is, $Q>Q_{0}$, then the optimal price $p^{*}(t, Q)$ just maximizes the instantaneous profit $[p(t)-c(t)] D(p(t), t)$ at each time $t$ and $Q-Q_{0}$ products are left for salvage at the end of horizon. If $Q_{1} \leq Q \leq Q_{0}$, then the products are just sold out at the end of the horizon. If $Q<Q_{1}$, then the product has been sold out before the end of the sales horizon. Moreover, the optimal price at each time is decreasing in the initial inventory level. That is, the higher the initial inventory level, the more the total sales quantity during the sales horizon.

3.2. Optimal Ordering Quantity. Based on the structure of the optimal pricing policy obtained in the previous section, we will determine the optimal ordering quantity in this section. Because the optimal pricing policy can be completely determined by the initial inventory level, the retailer's problem is to maximize its expected profit by choosing an appropriate ordering quantity. As mentioned in Section 2, the optimal ordering quantity can be determined by

$$
q^{*}=\arg \max _{q \geq 0}\left\{E J\left(p^{*}(\cdot, q \xi), q \xi\right)-w q E \xi\right\}
$$

where $p^{*}(\cdot, q \xi)$ is the optimal pricing policy when the realization of ordering quantity $q$ is $\xi q$ at the beginning of the horizon. For any realization of $\xi q$, we have obtained the closed form of optimal pricing policy $p^{*}(\cdot, q \xi)$ in Theorem 8 . Therefore, expression (46) is only dependent on the ordering quantity $q$, and we can determine the optimal ordering quantity by analyzing the characterization of expression (46). For notational simplicity, we define

$$
P(Q) \triangleq J\left(p^{*}(\cdot, Q), Q\right)-w Q, \quad Q \geq 0 .
$$


Then the optimal ordering quantity $q^{*}$ can be determined by

$$
q^{*}=\arg \max _{q \geq 0}\{E P(q \xi)\} \quad \text { or } \quad \int_{0}^{1} P^{\prime}\left(q^{*} z\right) z d \Psi(z)=0 .
$$

Note that $(E P(q \xi))^{\prime \prime}=E P^{\prime \prime}(q \xi) \xi^{2}$ and $\varphi(z)>0$ for $0 \leq$ $z \leq 1$, then we can obtain that if $P(Q)$ is concave on $[0, \infty)$ and strictly concave on an interval $\left[0, a_{0}\right)$, then $E P(q \xi)$ is strictly concave in $q$, where $a_{0}$ is a positive number. If $E P(q \xi)$ is strictly concave in $q$, the optimal ordering quantity $q^{*}$ is not only unique but also can be obtained by solving (48). The following result not only states that $P(Q)$ is concave on interval $[0, \infty)$ and strictly concave on interval $\left[0, Q_{0}\right)$ but also presents the closed forms of $P^{\prime}(Q)$. Therefore, we can obtain the optimal ordering quantity $q^{*}$ by solving (48).

Proposition 9. $P(Q)$ is concave and strictly concave on the intervals $[0, \infty)$, and $\left[0, Q_{0}\right)$ respectively. Moreover, the maximizer of $P(Q)$ belongs to the interval $\left(Q_{1}, Q_{0}\right)$.

From Proposition 9, we obtain $P^{\prime}(Q)>0$ for $0 \leq Q<\widehat{Q}$ and $P^{\prime}(Q)<0$ for $Q>\widehat{Q}$. Note that $Q_{1}<\widehat{Q}<Q_{0}$, then from (48), the optimal ordering quantity has to satisfy $q^{*}>\widehat{Q}$. Next, we discuss the optimal ordering quantity in two cases: $\widehat{Q}<q^{*} \leq Q_{0}$ and $q^{*}>Q_{0}$. Because $\int_{0}^{1} P^{\prime}\left(q^{*} z\right) z d \Psi(z)=0$ and $\int_{0}^{1} P^{\prime}(q z) z d \Psi(z)$ is strictly decreasing in $q$, we can derive the following conclusions. If $\int_{0}^{1} P^{\prime}\left(Q_{0} z\right) z d \Psi(z) \leq 0$, then we have $\widehat{Q}<q^{*} \leq Q_{0}$, and $q^{*}$ can be determined by

$$
\int_{0}^{\mathrm{Q}_{1} / q^{*}} P^{\prime}\left(q^{*} z\right) z d \Psi(z)+\int_{\mathrm{Q}_{1} / q^{*}}^{1} P^{\prime}\left(q^{*} z\right) z d \Psi(z)=0 .
$$

From (A.24) and (A.34), expression (49) can be written as

$$
\begin{aligned}
0=\int_{0}^{\mathrm{Q}_{1} / q^{*}}\left\{\frac{\alpha}{\beta} e^{-(a+\theta+\mu) t\left(q^{*} z\right)}+\frac{h}{a+\theta} e^{-(a+\theta) t\left(q^{*} z\right)}\right. \\
\left.-w-\frac{h}{a+\theta}\right\} z d \Psi(z) \\
+\int_{\mathrm{Q}_{1} / q^{*}}^{1}\left\{-\frac{q^{*} z-\gamma_{1}(T)}{e^{\theta T} \gamma_{2}(T)}+v e^{-(\theta+a) T}\right. \\
\left.-\frac{h}{\theta+a}\left[1-e^{-(\theta+a) T}\right]-w\right\} z d \Psi(z) .
\end{aligned}
$$

If $\int_{0}^{1} P^{\prime}\left(Q_{0} z\right) z d \Psi(z)>0$, then we have $q^{*}>Q_{0}$, and $q^{*}$ can be determined by

$$
\begin{gathered}
\int_{0}^{\mathrm{Q}_{1} / q^{*}} P^{\prime}\left(q^{*} z\right) z d \Psi(z)+\int_{\mathrm{Q}_{1} / q^{*}}^{\mathrm{Q}_{0} / q^{*}} P^{\prime}\left(q^{*} z\right) z d \Psi(z) \\
+\int_{\mathrm{Q}_{0} / q^{*}}^{1} P^{\prime}\left(q^{*} z\right) z d \Psi(z)=0 .
\end{gathered}
$$

From (A.24), (A.34), and (A.22), expression (51) can be written as

$$
\begin{aligned}
0= & \int_{0}^{\mathrm{Q}_{1} / q^{*}}\left\{\frac{\alpha}{\beta} e^{-(a+\theta+\mu) t\left(q^{*} z\right)}+\frac{h}{a+\theta} e^{-(a+\theta) t\left(q^{*} z\right)}\right. \\
& \left.-w-\frac{h}{a+\theta}\right\} z d \Psi(z) \\
& +\int_{\mathrm{Q}_{1} / q^{*}}^{\mathrm{Q}_{0} / q^{*}}\left\{-\frac{q^{*} z-\gamma_{1}(T)}{e^{\theta T} \gamma_{2}(T)}+v e^{-(\theta+a) T}\right. \\
& +\int_{\mathrm{Q}_{0} / q^{*}}^{1}\left\{v e^{-(\theta+a) T}-\frac{h}{\theta+a}\left[1-e^{-(\theta+a) T}\right]-w\right\} z d \Psi(z)
\end{aligned}
$$

Because $t(Q)$ does not have a closed form, we cannot obtain a closed form of the optimal ordering quantity $q^{*}$ by solving (50) and (52). Next, we present a numerical example to analyze the effects of the parameters on the optimal ordering quantity.

3.3. Numerical Example. In this section, we mainly concentrate on analyzing the optimal ordering quantity by a numerical example in which the random yield is normally distributed, that is, $\xi \sim N(m, d)$. Following Agrawal and Nahmias [14], we also assume that $0 \leq m \pm 3 d \leq 1$. Such assumption can guarantee that $\xi$ is between 0 and 1 with probability exceeding $99 \%$. The values of parameters in this example are given as follows: $\alpha=50, \beta=2, \theta=0.01$, $h=0.05, w=1, T=20$, and $v=0.2$. In Tables 1 and 2, Figures 1 and 2 , we present the effects of parameters $d, a, \mu$, and $m$ on the optimal ordering quantity, respectively. In Table 1, we set $a=0.005, \mu=0.05$ and $m=0.6$. From Table 1 , we can see that the optimal ordering quantity is first decreasing in $d$ and then increasing in $d$. This result implies that the optimal ordering quantity under deterministic yield is not necessarily less than the optimal ordering quantity under random yield. In Table 2 , we set $d=0.1, \mu=0.05$, and $m=0.7$. From Table 2, we can see that $q^{*}, Q_{0}$ and $Q_{1}$ are all increasing in $a$. That is, the larger the deterioration factor, the more the optimal ordering quantity. In Figure 1, we set $d=0.1, a=0.005$, and $m=0.7$. From Figure 1 , we can see that the optimal ordering quantity is decreasing in $\mu$. This result can be interpreted by the fact that the demand rate is decreasing in $\mu$ and the optimal ordering quantity is in direct proportion to the total demand during the horizon. From Figure 1, we imply that if the customers prefer to purchase fresher products, then the retailer should order less products. In Figure 2, we set $\mu=0.05, a=0.005$, and $d=0.1$. From Figure 2, we see that the optimal ordering quantity is decreasing in $m$. That is, the larger the mean of random yield, the less the optimal ordering quantity. This result is intuitive. 
TABLE 1: Effect of $d$ on the optimal ordering quantity.

\begin{tabular}{cccccccc}
\hline$d$ & 0.04 & 0.06 & 0.08 & 0.1 & 0.12 & 0.14 & 0.16 \\
\hline$q^{*}$ & 759.3 & 754.1 & 750.6 & 749.1 & 749.3 & 750.3 & 752.2 \\
\hline
\end{tabular}

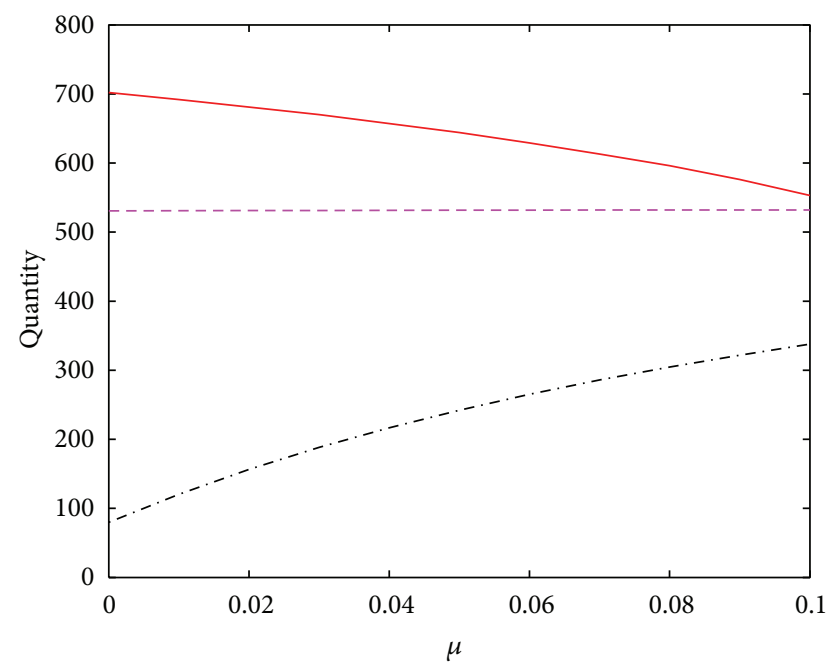

- Optimal order quantity $q^{*}$

- - Critical point $Q_{0}$

-. Critical point $Q_{1}$

FIGURE 1: Effect of $\mu$ on the optimal ordering quantity.

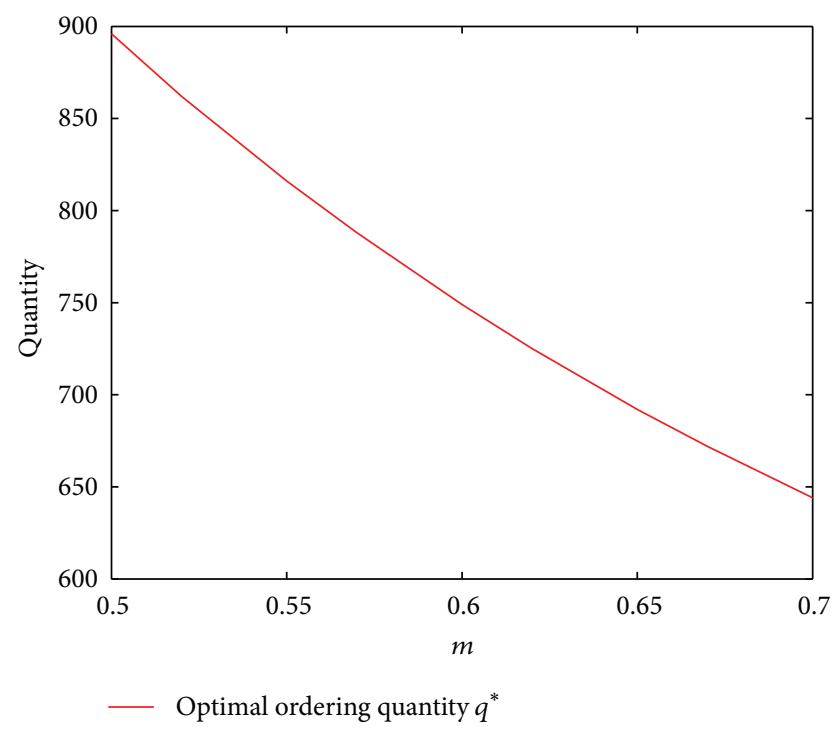

Figure 2: Effect of $m$ on the optimal ordering quantity.

\section{Conclusion}

In this paper, we have studied an EOQ model for a perishable product with random yield, price- and time-sensitive demand. First, by transforming the primal problem into a standard control problem, we present a general way to analyze the optimal pricing policy and the optimal ordering quantity. Second, we use Pontryagin's maximum principle to analyze a special case and obtain the structure of the optimal pricing policy. Based on the structure of the optimal pricing policy, we can obtain the optimal ordering quantity by solving an equation. Finally, we do some sensitivity analysis about the optimal ordering quantity with parameters $\mu, a$, $m$, and $d$ by a numerical example. From the numerical example, we obtain several intuitive results. For example, the more the customers prefer to purchase fresh products, the less the retailer orders products before the horizon and the larger the probability that the retailer sets higher price. One potential research direction is to study the optimal policies on stochastic demand, for example, the demand process can be assumed to be a diffusion process with price control. Another potential research direction is to study the situation in which the retailer has several ordering opportunity over a fixed horizon.

\section{Appendix}

Proof of Proposition 1. Substituting (4) and (5) into (2) and simplifying, we can obtain the following expression:

$$
\begin{aligned}
J(p(\cdot), Q)= & \int_{0}^{T} e^{-\theta t} p(t) D(p(t), t) d t \\
& -\int_{0}^{T} v e^{-\theta T} e^{-\int_{t}^{T} a(u) d u} D(p(t), t) d t \\
& +\int_{0}^{T} h e^{-\theta t} e^{-\int_{0}^{t} a(u) d u} \int_{0}^{t} e^{\int_{0}^{s} a(u) d u} D(p(s), s) d s d t \\
& +v Q e^{-\theta T-\int_{0}^{T} a(u) d u}-h Q \int_{0}^{T} e^{-\theta t-\int_{0}^{t} a(u) d u} d t
\end{aligned}
$$

According to integration by parts, the third part of the right hand of expression (A.1) can be transformed into the following expression:

$$
\int_{0}^{T} \int_{s}^{T} h e^{-\theta t} e^{-\int_{0}^{t} a(u) d u} e^{\int_{0}^{s} a(u) d u} D(p(s), s) d t d s .
$$

Substituting (A.2) into (A.1) and calculating, we have

$$
\begin{aligned}
& J(p(\cdot), Q) \\
& =\int_{0}^{T} e^{-\theta t}\left[p(t)-v e^{-\theta T+\theta t-\int_{t}^{T} a(u) d u}\right. \\
& \left.+h \int_{t}^{T} e^{\theta(t-s)-\int_{t}^{s} a(u) d u} d s\right] D(p(t), t) d t \\
& +v Q e^{-\theta T-\int_{0}^{T} a(u) d u}-h Q \int_{0}^{T} e^{-\theta t-\int_{0}^{t} a(u) d u} d t .
\end{aligned}
$$

With the help of (7) and (8), we can derive the result. 
TABLE 2: Effect of $a$ on the optimal ordering quantity.

\begin{tabular}{lccccccc}
\hline$a$ & 0.001 & 0.003 & 0.005 & 0.007 & 0.01 & 0.012 & 0.015 \\
\hline$q^{*}$ & 624 & 634 & 644 & 654 & 670 & 680 & 597 \\
\hline$Q_{0}$ & 510.8301 & 521.0969 & 531.6423 & 542.4748 & 559.2811 & 570.8704 & 588.8557 \\
\hline$Q_{1}$ & 226.2675 & 234.16 & 242.1696 & 250.3023 & 262.7455 & 271.213 & 284.1886 \\
\hline
\end{tabular}

Proof of Proposition 3. Solving (17), we obtain $\lambda(t)=$ $\lambda(T) e^{-\theta(T-t)}$. Substituting $\lambda(t)=\lambda(T) e^{-\theta(T-t)}$ into (15) and simplifying, we have

$$
H(p, \lambda, t)=\left[p-c(t)+\lambda(T) e^{-\theta(T-t)} e^{\int_{0}^{t} a(u) d u}\right] D(p, t) .
$$

Note that $H(p, \lambda, t)$ is concave in $p$ and $0 \leq p \leq \bar{p}$, then from (18), the optimal control is given by $\widetilde{p}(t, \lambda(T))$, which is defined as (19). Note that $\lambda(T) \leq 0$ in the optimal situation, we discuss the optimal pricing policy in two cases: $\lambda(T)=0$ and $\lambda(T)<0$. Suppose that $\lambda(T)=0$ in the optimal situation, then the optimal control is $\widetilde{p}(t, 0)$. Substituting $\widetilde{p}(t, 0)$ into $y(T)$, we derive

$$
y(T)=\int_{0}^{T} D(\widetilde{p}(s, 0), s) e^{\int_{0}^{s} a(u) d u} d s .
$$

(1) If $\int_{0}^{T} D(\widetilde{p}(s, 0), s) e^{\int_{0}^{s} a(u) d u} d s \leq Q$, then $\lambda(T)=0$ satisfies the sufficient conditions and $\widetilde{p}(t, 0)$ is just the optimal pricing policy. (2) If $\int_{0}^{T} D(\widetilde{p}(s, 0), s) e^{\int_{0}^{s} a(u) d u} d s>Q$, then $\lambda(T)=0$ is not feasible in the optimal situation. That is, $\lambda(T)<0$ and $y(T)=Q$ in the optimal situation. Note that $\widetilde{p}(t, \lambda(T))$, defined as (19), is the optimal pricing policy, then we can determine the value of $\lambda(T)$ by solving equation $y(T)=Q$, that is,

$$
\int_{0}^{T} D(\widetilde{p}(s, \lambda(T)), s) e^{\int_{0}^{s} a(u) d u} d s=Q .
$$

After determining $\lambda(T)$ by (A.6), we can obtain the optimal pricing policy $\widetilde{p}(t, \lambda(T))$.

Proof of Lemma 6. Taking derivatives of $g(t)$ with respect to $t$, we derive

$$
\begin{aligned}
g^{\prime}(t)= & -\frac{\alpha \mu}{2 \beta} e^{-\mu t}+\left[\frac{v(\theta+a)}{2} e^{-(\theta+a) T}+\frac{h}{2} e^{-(\theta+a) T}\right] e^{(\theta+a) t}, \\
g^{\prime \prime}(t)= & \frac{\alpha \mu^{2}}{2 \beta} e^{-\mu t} \\
& +\left[\frac{v(\theta+a)^{2}}{2} e^{-(\theta+a) T}+\frac{h(\theta+a)}{2} e^{-(\theta+a) T}\right] e^{(\theta+a) t}>0 .
\end{aligned}
$$

Then $g(t)$ is strictly convex in $t$. The solution to equation $g^{\prime}(t)=0$ can be described as

$$
t_{0}=\frac{1}{a+\theta+\mu} \ln \frac{\alpha \mu}{\beta v(\theta+a) e^{-(\theta+a) T}+\beta h e^{-(\theta+a) T}},
$$

and the minimum of $g(t)$ can be described as

$$
\begin{aligned}
g\left(t_{0}\right)= & {\left[\frac{\alpha}{2 \beta}+\frac{\alpha \mu}{2 \beta(\theta+a)}\right] } \\
& \times\left(\frac{\beta v(\theta+a) e^{-(\theta+a) T}+\beta h e^{-(\theta+a) T}}{\alpha \mu}\right)^{\mu /(a+\theta+\mu)} \\
& -\frac{h}{2(\theta+a)}
\end{aligned}
$$

where $t_{0}$ is the minimizer of $g(t)$. Because

$$
\begin{gathered}
g(T)=\frac{\alpha}{2 \beta} e^{-\mu T}+\frac{v}{2}>0, \\
g(0)=\frac{\alpha}{2 \beta}+\frac{v}{2} e^{-(\theta+a) T}+\frac{h}{2(\theta+a)}\left[1-e^{-(\theta+a) T}\right]>0,
\end{gathered}
$$

we derive the following conclusion: if $t_{0} \leq 0$ or $t_{0} \geq T$, then $g(t)>0$ for all $t \in[0, T]$; if $0<t_{0}<T$ and $g\left(t_{0}\right) \geq 0$, then $g(t) \geq 0$ for all $t \in[0, T]$.

Proof of Lemma 7. Because $g(t) \geq 0$ for all $t \in[0, T]$, the optimal pricing policy can be written as

$$
\widetilde{p}(t, \lambda(T))=\min \left\{f(t, \lambda(T)), \frac{\alpha}{\beta} e^{-\mu t}\right\} .
$$

In order to simplify the optimal pricing policy (A.11), we analyze the solution to the equation $f(t, \lambda(T))=(\alpha / \beta) e^{-\mu t}$, that is,

$$
\begin{gathered}
{\left[\frac{v}{2} e^{-(\theta+a) T}+\frac{h}{2(\theta+a)} e^{-(\theta+a) T}-\frac{\lambda(T)}{2} e^{-\theta T}\right] e^{(\theta+a) t}} \\
=\frac{\alpha}{2 \beta} e^{-\mu t}+\frac{h}{2(\theta+a)}
\end{gathered}
$$

From (A.12), we can obtain the following conclusions: (1) If $\lambda(T) \geq v e^{-a T}+(h /(\theta+a)) e^{-a T}$, then (A.12) has no solution, and the optimal pricing policy can be described as $\tilde{p}(t, \lambda(T))=f(t, \lambda(T))$ for all $t \in[0, T]$. (2) If $v e^{-a T}-$ $(\alpha / \beta) e^{-(a+\mu) T}<\lambda(T)<v e^{-a T}+(h /(\theta+a)) e^{-a T}$, then (A.12) has a unique solution on interval $(T, \infty)$, and the optimal pricing policy can be described as $\widetilde{p}(t, \lambda(T))=f(t, \lambda(T))$ for all $t \in[0, T]$. (3) If $-(\alpha / \beta) e^{\theta T}+v e^{-a T}-(h /(\theta+$ a)) $\left(e^{\theta T}-e^{-a T}\right)<\lambda(T) \leq v e^{-a T}-(\alpha / \beta) e^{-(a+\mu) T}$, then (A.12) has a unique solution $t(\lambda(T))$ on interval $(0, T]$, and 
the optimal pricing policy can be described as $\widetilde{p}(t, \lambda(T))=$ $f(t, \lambda(T))$ for $t \in[0, t(\lambda(T))]$ and $\tilde{p}(t, \lambda(T))=(\alpha / \beta) e^{-\mu t}$ for $t \in(t(\lambda(T)), T]$. (4) If $\lambda(T) \leq-(\alpha / \beta) e^{\theta T}+v e^{-a T}-(h /(\theta+$ a) $)\left(e^{\theta T}-e^{-a T}\right)$, then (A.12) has a unique solution on interval $(-\infty, 0]$, and the optimal pricing policy can be described as $\widetilde{p}(t, \lambda(T))=(\alpha / \beta) e^{-\mu t}$ for all $t \in[0, T]$. In other words, the retailer will not order and sell the product in this case. Because $\lambda(T) \leq 0$ in the optimal situation and $v e^{-a T}+(h /(\theta+$ a)) $e^{-a T}>0$, the result holds.

Proof of Theorem 8. From Lemma 7, we have known that if $v e^{-a T}-(\alpha / \beta) e^{-(a+\mu) T}<\lambda(T) \leq 0$, the total sales quantity during the whole horizon can be described as

$$
\begin{aligned}
Q(\lambda(T)) & =\int_{0}^{T}\left(\alpha-\beta e^{\mu t} p^{*}(t, \lambda(T))\right) e^{a t} d t \\
& =\int_{0}^{T}\left(\frac{\alpha}{2}-\frac{\beta}{2} e^{\mu t} c(t)+\frac{\beta}{2} \lambda(T) e^{(a+\theta+\mu) t-\theta T}\right) e^{a t} d t \\
& =\gamma_{1}(T)+\gamma_{2}(T) \lambda(T)
\end{aligned}
$$

Note that $v e^{-a T}-(\alpha / \beta) e^{-(a+\mu) T}<\lambda(T) \leq 0$ and $\gamma_{2}(T)>0$, then the total sales quantity, denoted as (A.13), satisfies the following expression:

$$
Q_{1}=Q\left(v e^{-a T}-\frac{\alpha}{\beta} e^{-(a+\mu) T}\right)<Q(\lambda(T)) \leq Q(0)=Q_{0} .
$$

Therefore, if $Q_{1}<Q \leq Q_{0}$, there exists a unique $\lambda(T)=(Q-$ $\left.\gamma_{1}(T)\right) / \gamma_{2}(T) \in\left(v e^{-a T}-(\alpha / \beta) e^{-(a+\mu) T}, 0\right]$ such that $Q(\lambda(T))=$ $Q$ and $\lambda(T)=\left(Q-\gamma_{1}(T)\right) / \gamma_{2}(T)$ is optimal. From Lemma 7, the optimal pricing policy can be described as (42). If $Q>Q_{0}$, then the optimal value of $\lambda(T)$ must be zero, and the total sales quantity is $Q_{0}$. If $\lambda(T) \leq v e^{-a T}-(\alpha / \beta) e^{-(a+\mu) T}$, then from Lemma 7 , the total sales quantity during the horizon is

$$
\begin{aligned}
Q(\lambda(T)) & =\int_{0}^{t(\lambda(T))}\left(\alpha-\beta e^{\mu t} p^{*}(t, \lambda(T))\right) e^{a t} d t \\
& =\int_{0}^{t(\lambda(T))}\left(\frac{\alpha}{2}-\frac{\beta}{2} e^{\mu t} c(t)+\frac{\beta}{2} \lambda(T) e^{(a+\theta+\mu) t-\theta T}\right) e^{a t} d t \\
& =\gamma_{1}(t(\lambda(T)))+\gamma_{2}(t(\lambda(T))) \lambda(T) .
\end{aligned}
$$

From expression (A.12), $t(\lambda(T))$ is the unique solution to the equation

$$
\begin{aligned}
\lambda(T)= & {\left[v e^{-(\theta+a) T}+\frac{h}{\theta+a} e^{-(\theta+a) T}\right.} \\
& \left.-\frac{\alpha}{\beta} e^{-(a+\theta+\mu) t(\lambda(T))}-\frac{h}{\theta+a} e^{-(\theta+a) t(\lambda(T))}\right] e^{\theta T} .
\end{aligned}
$$

Substituting (A.16), (39) into (A.15) and simplifying, the total sales quantity can be written as

$$
\begin{aligned}
Q( & t(\lambda(T))) \\
= & \frac{\alpha}{2 a}\left(e^{a t(\lambda(T))}-1\right)+\frac{\beta h}{2(\theta+a)(a+\mu)}\left[e^{(a+\mu) t(\lambda(T))}-1\right] \\
& -\frac{\beta}{2(2 a+\theta+\mu)}\left(e^{(2 a+\theta+\mu) t(\lambda(T))}-1\right) \\
& \times\left[\frac{\alpha}{\beta} e^{-(a+\theta+\mu) t(\lambda(T))}+\frac{h}{\theta+a} e^{-(\theta+a) t(\lambda(T))}\right] .
\end{aligned}
$$

Taking derivative of $Q(\lambda(T))$ with respect to $t(\lambda(T))$ and simplifying, we derive

$$
\begin{aligned}
& \frac{d Q(t(\lambda(T)))}{d t(\lambda(T))} \\
& =\frac{\beta}{2(2 a+\theta+\mu)}\left(e^{(2 a+\theta+\mu) t(\lambda(T))}-1\right) \\
& \quad \times\left[(a+\theta+\mu) \frac{\alpha}{\beta} e^{-(a+\theta+\mu) t(\lambda(T))}+h e^{-(\theta+a) t(\lambda(T))}\right] .
\end{aligned}
$$

Then $Q(t(\lambda(T)))$ is increasing in $t(\lambda(T))$. Note that $0<$ $t(\lambda(T)) \leq T$, then we have

$$
0=Q(0)<Q(t(\lambda(T))) \leq Q(T)=Q_{1} .
$$

Therefore, if $0<Q \leq Q_{1}$, there exists a unique $\lambda(T)=$ $\left(Q-\gamma_{1}(t(Q))\right) / \gamma_{2}(t(Q)) \leq v e^{-a T}-(\alpha / \beta) e^{-(a+\mu) T}$ such that the total sales quantity is $Q$, and the optimal pricing policy can be described as (43), where $t(Q)$ is defined as

$$
\begin{aligned}
Q= & \frac{\alpha}{2 a}\left(e^{a t(\mathrm{Q})}-1\right)+\frac{\beta h}{2(\theta+a)(a+\mu)}\left[e^{(a+\mu) t(\mathrm{Q})}-1\right] \\
& -\frac{\beta}{2(2 a+\theta+\mu)}\left(e^{(2 a+\theta+\mu) t(\mathrm{Q})}-1\right) \\
& \times\left[\frac{\alpha}{\beta} e^{-(a+\theta+\mu) t(\mathrm{Q})}+\frac{h}{\theta+a} e^{-(\theta+a) t(\mathrm{Q})}\right] .
\end{aligned}
$$

Proof of Proposition 9. Based on the optimal pricing policy obtained in Theorem 8 , we prove the concavity of $P(Q)$ in three cases: $Q>Q_{0}, Q_{1} \leq Q \leq Q_{0}$, and $0 \leq Q<Q_{1}$. If $Q>Q_{0}$, then from Theorem 8, (6), and (26), $P(Q)$ can be described as

$$
\begin{aligned}
P(Q)= & \int_{0}^{T} e^{-\theta t}\left[p^{*}(t, Q)-c(t)\right]\left[\alpha-\beta e^{\mu t} p^{*}(t, Q)\right] d t \\
& +A-w Q
\end{aligned}
$$




$$
\begin{aligned}
& =\int_{0}^{T} e^{-\theta t}\left\{\frac{e^{-\mu t}}{\beta}\left[\frac{\alpha}{2}+\frac{\beta}{2} e^{\mu t} c(t)\right]^{2}-\alpha c(t)\right\} d t+A-w Q \\
& =\gamma_{3}(T)+v e^{-(\theta+a) T} Q-\frac{h Q}{\theta+a}\left[1-e^{-(\theta+a) T}\right]-w Q,
\end{aligned}
$$

where $\gamma_{3}(T)$ is defined as (40). Taking derivative of $P(Q)$ with respect to $Q$, we derive

$$
P^{\prime}(Q)=v e^{-(\theta+a) T}-\frac{h}{\theta+a}\left[1-e^{-(\theta+a) T}\right]-w<0 .
$$

Then $P(Q)$ is linearly decreasing in $Q$ on the interval $\left(Q_{0}, \infty\right)$. Therefore, $P(Q)$ is concave in $Q$ on the interval $\left(Q_{0}, \infty\right)$. If $Q_{1} \leq Q \leq Q_{0}$, then from Theorem 8, (6), and (26), $P(Q)$ can be described as

$$
\begin{aligned}
P(Q)= & \int_{0}^{T} e^{-\theta t}\left[p^{*}(t, Q)-c(t)\right]\left[\alpha-\beta e^{\mu t} p^{*}(t, Q)\right] d t \\
& +A-w Q \\
= & -\left(\frac{Q-\gamma_{1}(T)}{\gamma_{2}(T)}\right)^{2} \int_{0}^{T} \frac{\beta}{4} e^{(2 a+\mu+\theta) t-2 \theta T} d t \\
& +\int_{0}^{T} e^{-\theta t}\left\{\frac{e^{-\mu t}}{\beta}\left[\frac{\alpha}{2}+\frac{\beta}{2} e^{\mu t} c(t)\right]^{2}-\alpha c(t)\right\} d t+A-w Q \\
= & -\frac{\left[Q-\gamma_{1}(T)\right]^{2}}{2 e^{\theta T} \gamma_{2}(T)}+\gamma_{3}(T)+v e^{-(\theta+a) T} Q \\
& -\frac{h Q}{\theta+a}\left[1-e^{-(\theta+a) T}\right]-w Q .
\end{aligned}
$$

Taking derivative of $P(Q)$ with respect to $Q$, we derive

$$
\begin{aligned}
\frac{d P(Q)}{d Q}= & -\frac{Q-\gamma_{1}(T)}{e^{\theta T} \gamma_{2}(T)}+v e^{-(\theta+a) T} \\
& -\frac{h}{\theta+a}\left[1-e^{-(\theta+a) T}\right]-w .
\end{aligned}
$$

Then $P(Q)$ is strictly concave in $Q$ on the interval $\left[Q_{1}, Q_{0}\right]$. From (A.13) and (A.14), we have

$$
\frac{Q_{0}-\gamma_{1}(T)}{\gamma_{2}(T)}=0, \quad \frac{Q_{1}-\gamma_{1}(T)}{\gamma_{2}(T)}=v e^{-a T}-\frac{\alpha}{\beta} e^{-(a+\mu) T} .
$$

Thus, from (A.24) and (A.25), we have

$$
\begin{aligned}
\left.\frac{d P(Q)}{d Q}\right|_{\mathrm{Q}=\mathrm{Q}_{1}}= & -\frac{1}{e^{\theta T}}\left[v e^{-a T}-\frac{\alpha}{\beta} e^{-(a+\mu) T}\right] \\
& +v e^{-(\theta+a) T}-\frac{h}{\theta+a}\left[1-e^{-(\theta+a) T}\right]-w \\
= & \frac{\alpha}{\beta} e^{-(a+\theta+\mu) T}-\frac{h}{\theta+a}\left[1-e^{-(\theta+a) T}\right]-w, \\
\left.\frac{d P(Q)}{d Q}\right|_{\mathrm{Q}=\mathrm{Q}_{1}}= & v e^{-(\theta+a) T}-\frac{h}{\theta+a}\left[1-e^{-(\theta+a) T}\right]-w .
\end{aligned}
$$

From (A.22) and (A.27), we derive $\lim _{\mathrm{Q} \rightarrow \mathrm{Q}_{0}{ }^{+}} P^{\prime}(\mathrm{Q})=$ $\lim _{\mathrm{Q} \rightarrow \mathrm{Q}_{0}-} P^{\prime}(Q)$. Then $P(Q)$ is concave on the interval $\left[Q_{1}, \infty\right)$. If $0 \leq Q<Q_{1}$, then from Theorem 8, (6), and (26), $P(Q)$ can be described as

$$
\begin{aligned}
P(Q)= & \int_{0}^{t(Q)} e^{-\theta t}\left[p^{*}(t, Q)-c(t)\right] \\
& \times\left[\alpha-\beta e^{\mu t} p^{*}(t, Q)\right] d t+A-w Q \\
= & -\frac{\left[Q-\gamma_{1}(t(Q))\right]^{2}}{2 e^{\theta T} \gamma_{2}(t(Q))}+\gamma_{3}(t(Q)) \\
& +\left(v e^{-(\theta+a) T}-\frac{h}{\theta+a}\left[1-e^{-(\theta+a) T}\right]-w\right) Q,
\end{aligned}
$$

where $t(Q)$ is defined as (A.20). Taking derivative on both sides of expression (A.20) with respect to $Q$ and simplifying, we obtain

$$
\begin{aligned}
\frac{d t(Q)}{d Q}= & \frac{2(2 a+\theta+\mu)}{\beta}\left[e^{(2 a+\theta+\mu) t(\mathrm{Q})}-1\right]^{-1} \\
& \times\left[\frac{\alpha}{\beta}(a+\theta+\mu) e^{-(a+\theta+\mu) t(\mathrm{Q})}+h e^{-(a+\theta) t(\mathrm{Q})}\right]^{-1} .
\end{aligned}
$$

From Theorem 8 and (44), we have

$$
\begin{aligned}
\lambda(T)= & \frac{Q-\gamma_{1}(t(Q))}{\gamma_{2}(t(Q))} \\
= & {\left[v e^{-(\theta+a) T}+\frac{h}{\theta+a} e^{-(\theta+a) T}-\frac{\alpha}{\beta} e^{-(a+\theta+\mu) t(Q)}\right.} \\
& \left.\quad-\frac{h}{\theta+a} e^{-(\theta+a) t(Q)}\right] e^{\theta T} .
\end{aligned}
$$

Then expression (A.28) can be written as

$$
\begin{aligned}
P(Q)= & -\frac{1}{2 e^{\theta T}} \lambda^{2}(T) \gamma_{2}(t(Q))+\gamma_{3}(t(Q)) \\
& +\left(v e^{-(\theta+a) T}-\frac{h}{\theta+a}\left[1-e^{-(\theta+a) T}\right]-w\right) Q,
\end{aligned}
$$

and the first derivative of $\lambda(T)$ with respect to $Q$ can be described as

$$
\begin{array}{r}
\frac{d \lambda(T)}{d Q}=-e^{\theta T}\left[(a+\theta+\mu) \frac{\alpha}{\beta} e^{-(a+\theta+\mu) t(\mathrm{Q})}\right. \\
\left.+h e^{-(\theta+a) t(Q)}\right] \frac{d t(Q)}{d Q} .
\end{array}
$$


From the definition of $\gamma_{3}(x)$, we have

$$
\begin{aligned}
\frac{d \gamma_{3}(x)}{d x}= & {\left[\frac{\beta v^{2}}{4} e^{-2(\theta+a) T}+\frac{\beta h^{2}}{4(\theta+a)^{2}} e^{-2(\theta+a) T}\right.} \\
& \left.+\frac{\beta v h}{2(\theta+a)} e^{-(2 a+2 \theta) T}\right] e^{(2 a+\theta+\mu) x} \\
& -\left[\frac{\beta h^{2}}{2(\theta+a)^{2}} e^{-(\theta+a) T}+\frac{\beta v h}{2(\theta+a)} e^{-(\theta+a) T}\right] e^{(a+\mu) x} \\
& +\frac{\beta h^{2}}{4(\theta+a)^{2}} e^{(\mu-\theta) x} \\
& -\left[\frac{\alpha v}{2} e^{-(\theta+a) T}+\frac{\alpha h}{2(\theta+a)} e^{-(\theta+a) T}\right] e^{a x} \\
& +\frac{\alpha h}{2(a+\theta)} e^{-\theta x}+\frac{\alpha^{2}}{4 \beta} e^{-(\theta+\mu) x} .
\end{aligned}
$$

Therefore, by taking derivative on both sides of expression (A.31) with respect to $Q$ and simplifying, we have

$$
\begin{aligned}
\frac{d P(Q)}{d Q}= & -\frac{\lambda(T)}{e^{\theta T}} \gamma_{2}(t(Q)) \frac{d \lambda(T)}{d Q} \\
& -\left[\frac{1}{2 e^{\theta T}} \lambda^{2}(T) \gamma_{2}^{\prime}(t(Q))-\gamma_{3}^{\prime}(t(Q))\right] \frac{d t(Q)}{d Q} \\
& +v e^{-(\theta+a) T}-\frac{h}{\theta+a}\left[1-e^{-(\theta+a) T}\right]-w \\
= & \frac{\alpha}{\beta} e^{-(a+\theta+\mu) t(\mathrm{Q})}+\frac{h}{a+\theta} e^{-(a+\theta) t(Q)}-w-\frac{h}{a+\theta} .
\end{aligned}
$$

From (A.29), we obtain that $t(Q)$ is strictly increasing in $Q$. Therefore, $d P(Q) / d Q$ is strictly decreasing in $Q$ on the interval $\left[0, Q_{1}\right)$. That is, $P(Q)$ is strictly concave in $Q$ on the interval $\left[0, Q_{1}\right)$. From (A.19), we have $t\left(Q_{1}\right)=T$. Therefore, from (A.34), we derive

$$
\begin{aligned}
\left.\frac{d P(Q)}{d Q}\right|_{\mathrm{Q}=\mathrm{Q}_{1}}= & \frac{\alpha}{\beta} e^{-(a+\theta+\mu) T}+\frac{h}{a+\theta} e^{-(a+\theta) T} \\
& -w-\frac{h}{a+\theta}>0,
\end{aligned}
$$

and $P(Q)$ is strictly increasing on the interval $\left[0, Q_{1}\right)$. From (A.26) and (A.35), we have $\lim _{Q \rightarrow Q_{1}+} P^{\prime}(Q)=$ $\lim _{Q \rightarrow Q_{1}-} P^{\prime}(Q)$. Hence, $P(Q)$ is concave and strictly concave on the intervals $[0, \infty)$ and $\left[0, Q_{0}\right)$, respectively. Because $P(Q)$ is strictly increasing on $\left[0, Q_{1}\right]$ and strictly decreasing on $\left[Q_{0}, \infty\right)$, the maximizer of $P(Q)$ belongs to the interval $\left(Q_{1}, Q_{0}\right)$. Moreover, from (A.24), the maximizer of $P(Q)$ can be described as

$$
\begin{aligned}
\widehat{Q}= & \gamma_{1}(T) \\
& +\left(v e^{-(\theta+a) T}-\frac{h}{\theta+a}\left[1-e^{-(\theta+a) T}\right]-w\right) e^{\theta T} \gamma_{2}(T) .
\end{aligned}
$$

Substituting (A.36) into (A.23) and simplifying, the maximum of $P(Q)$ can be described as

$$
\begin{aligned}
P(\widehat{Q})= & -\frac{\left[\widehat{Q}-\gamma_{1}(T)\right]^{2}}{2 e^{\theta T} \gamma_{2}(T)}+\gamma_{3}(T) \\
& +\left\{v e^{-(\theta+a) T}-\frac{h}{a+\theta}\left[1-e^{-(a+\theta) T}\right]-c\right\} \widehat{Q} .
\end{aligned}
$$

The proof is completed.

\section{Acknowledgments}

The authors would like to thank the editor and two anonymous referees, whose comments improved our work significantly. This work was partially supported by National Natural Science Foundation of China under Grant nos. 71201027 and 71272085, Humanity and Social Science Youth Foundation of Ministry of Education of China under Grant no. 12YJC630260, Guangdong Natural Science Foundation under Grant no. S2012040007919, foundation for Distinguished Young Talents in Higher Education of Guangdong under Grant no. LYM11121, and the Open Fund of Chongqing Key Laboratory of Logistics under Grant no. CQKLL12003.

\section{References}

[1] A. Latour, "Trial by fire: a blaze in Albuquerque sets off major crisis for cell-phone giants," Wall Street Journal. In press.

[2] Y. Sheffi, The Resilient Enterprise-Overcoming Vulnerability For Competitive Advantage, The MIT Press, Cambridge, Mass, USA, 2005.

[3] Y. Gerchak, R. G. Vickson, and M. Parlar, "Periodic review production models with variable yield and uncertain demand," IIE Transactions, vol. 20, no. 2, pp. 144-150, 1988.

[4] F. W. Ciarallo, R. Akella, and T. E. Morton, "A periodic review, production planning model with uncertain capacity and uncertain demand-optimality of extended myopic policies," Management Science, vol. 40, no. 3, pp. 320-332, 1994.

[5] Y.Z. Wang and Y. Gerchak, "Periodic review production models with variable capacity, random yield, and uncertain demand," Management Science, vol. 42, no. 1, pp. 130-137, 1996.

[6] A. S. Erdem and S. Özekici, "Inventory models with random yield in a random environment," International Journal of Production Economics, vol. 78, no. 3, pp. 239-253, 2002.

[7] Q. Li and S. H. Zheng, "Joint inventory replenishment and pricing control for systems with uncertain yield and demand," Operations Research, vol. 54, no. 4, pp. 696-705, 2006.

[8] X. Chao, H. Chen, and S. Zheng, "Joint replenishment and pricing decisions in inventory systems with stochastically dependent supply capacity," European Journal of Operational Research, vol. 191, no. 1, pp. 142-155, 2008.

[9] Q. Feng, "Integrating dynamic pricing and replenishment decisions under supply capacity uncertainty," Management Science, vol. 56, no. 12, pp. 2154-2172, 2010.

[10] A. S. Erdem, M. M. Fadilog̃lu, and S. Özekici, "An EOQ model with multiple suppliers and random capacity," Naval Research Logistics, vol. 53, no. 1, pp. 101-114, 2006. 
[11] M. M. Fadıloğlu, E. Berk, and M. Gürbüz, "Supplier diversification under binomial yield," Operations Research Letters, vol. 36, no. 5, pp. 539-542, 2008.

[12] X. Yan and K. Liu, "Effects of setup cost and random yield on the structure of optimal ordering policy," Journal of the Chinese Institute of Industrial Engineers, vol. 27, no. 1, pp. 52-60, 2010.

[13] M. M. Tajbakhsh, C. G. Lee, and S. Zolfaghari, "On the supplier diversification under binomial yield," Operations Research Letters, vol. 38, no. 6, pp. 505-509, 2010.

[14] N. Agrawal and S. Nahmias, "Rationalization of the supplier base in the presence of yield uncertainty," Production and Operations Management, vol. 6, no. 3, pp. 291-308, 1997.

[15] K. Inderfurth, "Analytical solution for a single-period production-inventory problem with uniformly distributed yield and demand," Central European Journal of Operations Research, vol. 12, no. 2, pp. 117-127, 2004.

[16] M. Dada, N. C. Petruzzi, and L. B. Schwarz, "A newsvendor's procurement problem when suppliers are unreliable," Manufacturing and Service Operations Management, vol. 9, no. 1, pp. 932, 2007.

[17] S. E. Grasman, Z. Sari, and T. Sari, "Newsvendor solutions with general random yield distributions," RAIRO Operations Research, vol. 41, no. 4, pp. 455-464, 2007.

[18] Y. Rekik, E. Sahin, and Y. Dallery, "A comprehensive analysis of the Newsvendor model with unreliable supply," OR Spectrum, vol. 29, no. 2, pp. 207-233, 2007.

[19] B. Maddah, M. K. Salameh, and G. M. Karame, "Lot sizing with random yield and different qualities," Applied Mathematical Modelling, vol. 33, no. 4, pp. 1997-2009, 2009.

[20] S. Y. Tang and P. Kouvelis, "Supplier diversification strategies in the presence of yield uncertainty and buyer competition," Manufacturing \& Service Operations Management, vol. 13, no. 4, pp. 439-451, 2011.

[21] A. Rajan, R. Steinberg, and R. Steinberg, "Dynamic pricing and ordering decisions by a monopolist," Management Science, vol. 38 , no. 2, pp. 240-262, 1992.

[22] P. L. Abad, "Optimal pricing and lot-sizing under conditions of perishablity and partial backordering," Management Science, vol. 42, no. 8, pp. 1093-1104, 1996.

[23] P. L. Abad, "Optimal price and order size for a reseller under partial backordering," Computers \& Operations Research, vol. 28, no. 1, pp. 53-65, 2001.

[24] J. T. Teng and C. T. Chang, "Economic production quantity models for deteriorating items with price- and stock-dependent demand," Computers \& Operations Research, vol. 32, no. 2, pp. 297-308, 2005.

[25] J. M. Chen and L. T. Chen, "Periodic pricing and replenishment policy for continuously decaying inventory with multivariate demand," Applied Mathematical Modelling, vol. 31, no. 9, pp. 1819-1828, 2007.

[26] S. W. Shinn and H. Hwang, "Optimal pricing and ordering policies for retailers under order-size-dependent delay in payments," Computers \& Operations Research, vol. 30, no. 1, pp. 3550, 2003.

[27] F. S. Chou and M. Parlar, "Optimal control of a revenue management system with dynamic pricing facing linear demand," Optimal Control Applications \& Methods, vol. 27, no. 6, pp. 323347, 2006.

[28] P. S. You and Y. C. Hsieh, "An EOQ model with stock and price sensitive demand," Mathematical and Computer Modelling, vol. 45, no. 7-8, pp. 933-942, 2007.
[29] L. M. A. Chan, Z. J. M. Shen, D. Simchi-Levi, and J. Swann, "Coordination of pricing and inventory decisions: a survey and classification," in Handbook of Quantitative Supply Chain Analysis: Modeling in the E-business Era, D. Simchi-Levi, S. D. Wu, and Z. J. M. Shen, Eds., pp. 335-392, Kluwer, Amsterdam, The Netherlands, 2004.

[30] W. Elmaghraby and P. Keskinocak, "Dynamic pricing in the presence of inventory considerations: research overview, current practices and future directions," Management Science, vol. 49, no. 10, pp. 1287-1309, 2003.

[31] S. P. Sethi and G. L. Thompson, Optimal Control Theory, Kluwer Academic Publishers, Boston, Mass, USA, 2nd edition, 2000, Applications to management science and economics. 


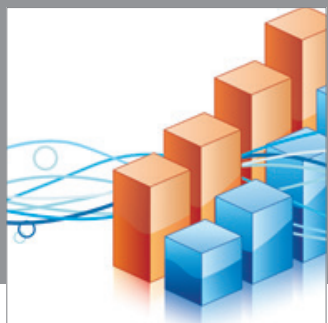

Advances in

Operations Research

mansans

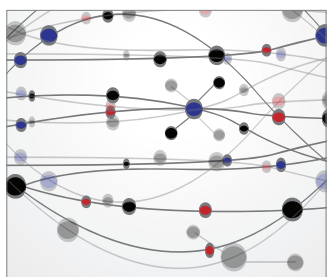

The Scientific World Journal
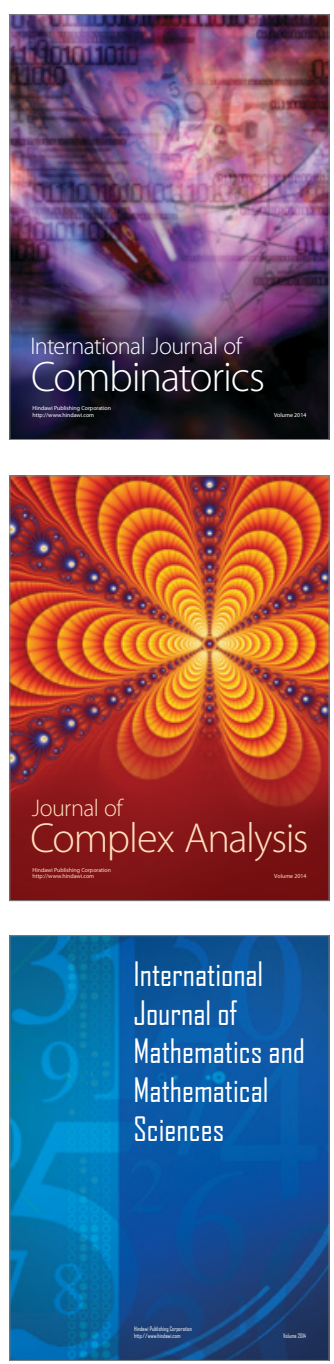
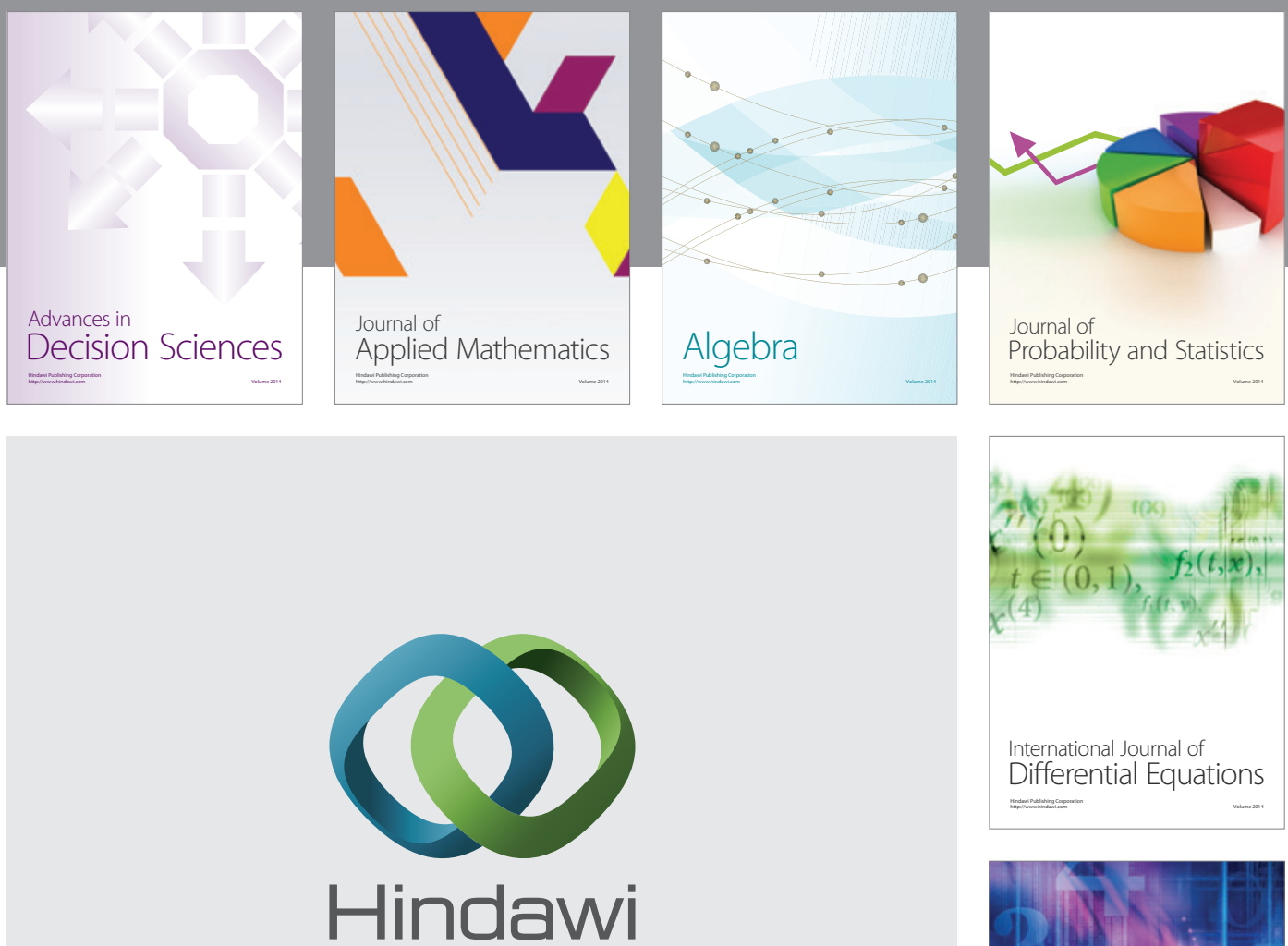

Submit your manuscripts at http://www.hindawi.com
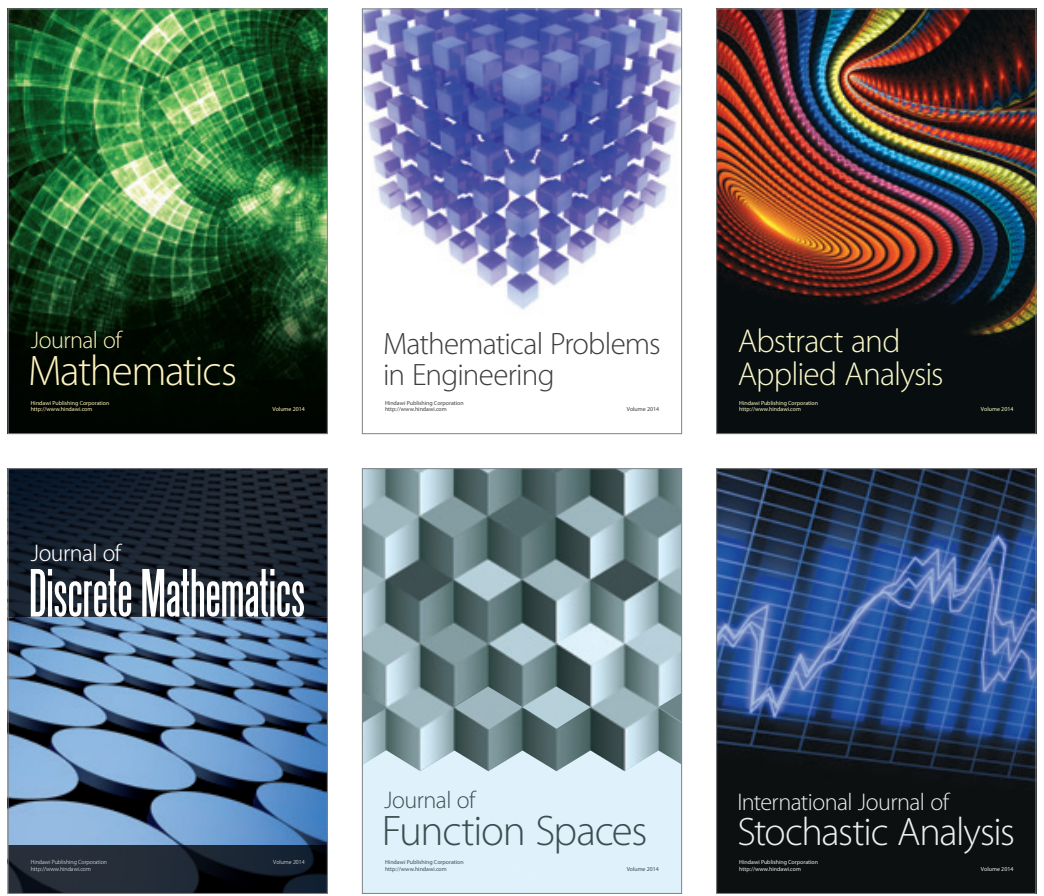

Journal of

Function Spaces

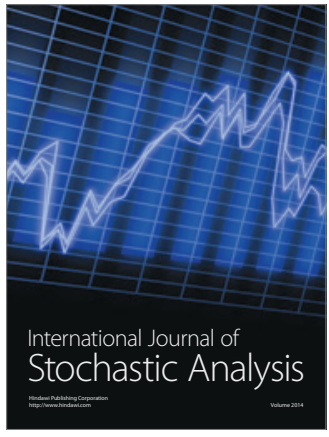

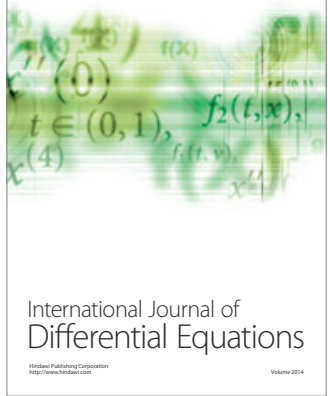
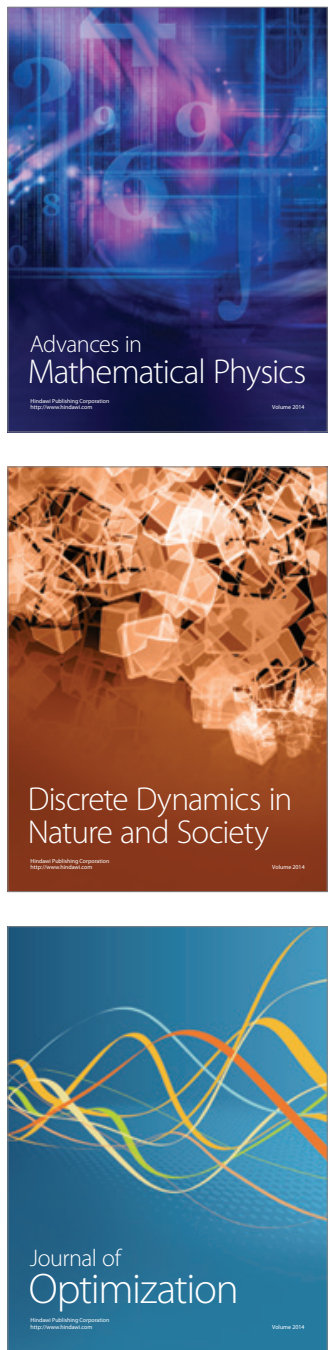